\title{
A Phenomenon in the Trigeminal System
}

\author{
HENRY H. YU and JAMES K. AVERY \\ Dental Research Institute and Department of Oral Biology, University of Michigan \\ School of Dentistry, Ann Arbor, Michigan 48104, USA
}

There is no anatomical evidence that a primary afferent nerve terminates directly on another primary afferent nerve. The simplest pathway responsible for primary afferent depolarization after peripheral nerve stimulation would involve at least two synapses. Physiological studies in the spinal cord generally support the anatomical findings, although recently Van Harreveld and Niechaj suggested that a monosynaptic com ponent of the dorsal root potential might exist (Brain Res 19: 105-116, 1970). In the trigeminal system, tight junction (HINRICHSEN and LARRAMENDI, Brain Res 7: 296-299, 1968) or electro tonic coupling (BAKER and Llinas, J Physiol 212 45-63, 1971) has been shown in the mesence phalic nucleus but has not been demonstrated in the trigeminal ganglion.

While attempting to record the intracellular events at the central preterminal of a trigeminal nerve fiber, we observed a phenomenon indicating unexpected interaction between the me dial and lateral branches of the infraorbital nerve. We used a conventional microelectrode technique. Stimuli were delivered to the medial or lateral branch of the infraorbital nerve after it emerged from the infraorbital foramen. Responses were recorded in the oral part of the spinal nucleus. When the lateral branch was stimulated, the latency of the fiber action poten-

This investigation was supported by LSPHS Research Grant DE 02731 to the Dental Research Institute, the University of Michigan, Ann Arbor, Mich.

Received for publication September 20, 1973. tial was 0.6 msec and the refractoriness, 0.2 msec; the spikes followed the stimulation up to 450 $\mathrm{Hz}$ (Illustration: $B, C$, and $F$ ). With a conduction distance of about $50 \mathrm{~mm}$, the short latency and frequency responses indicated that the re. cordings were being made at the preterminal of a lateral branch primary afferent fiber $(B)$. However, when the medial branch was stimulated, the latency was $0.8 \mathrm{msec}$ and the refractoriness, $0.8 \mathrm{msec}$; the recorded potentials did not completely respond to $90 \mathrm{~Hz}$ of stimulation ( $A$, $C$, and $E$ ). If the current had spread from the medial branch stimulating site to the nerve fiber where the recording actually was made, the responses would have been identical; however, the refractoriness and frequency responses were different.

There are three possible explanations for this phenomenon. First, a single nerve fiber from the lateral branch of the infraorbital nerve may send a smaller collateral fiber to the medial branch. The response of both branches of the single fiber to peripheral stimulation would be different, since the larger branch would conduct faster, respond to higher frequencies, and have a shorter refractory period. Second, some sort of synaptic contacts between primary afferent nerves might exist either at the terminals or at the ganglion level. Third, this might be a situation of ephaptic transmission due either to a naturally occurring electric coupling between cellular elements or an artificially created one. More experiments are needed to clarify the ambiguity of the phenomenon.

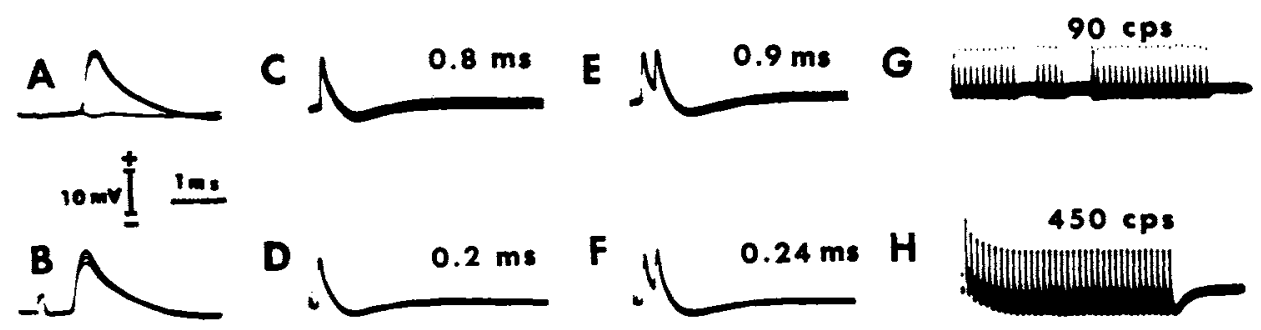

Responses recorded from central preterminal of infraorbital nerve fiber, $A, C, E$, and $G$ : medial branch of nerve was stimulated. $B, D, F$, and $H$ : lateral branch of nerve was stimulated. $A$ and $B$ : action potentials' response to suprathreshold single pulse stimulation. Flat line in $A$ indicates that response to subthreshold stimulation was superimposed. $C, D, E$, and $F$ show that responses resulted from double stimulation, with intervals indicated. $G$ and $H$ are responses following high frequency stimulation. 